Cahiers $d u$ MONDE RUSSE

\section{Cahiers du monde russe}

Russie - Empire russe - Union soviétique et États indépendants

42/2-4 | 2001

La police politique en Union soviétique, 1918-1953

\title{
The Great terror in the provinces of the USSR,
}

1937-1938

A cooperative bibliography

Rolf BINNER, Marc JUNG et Terry MARTIN

\section{CpenEdition}

Journals

Édition électronique

URL : https://journals.openedition.org/monderusse/8474

DOI : $10.4000 /$ monderusse. 8474

ISSN : $1777-5388$

Éditeur

Éditions de l'EHESS

Édition imprimée

Date de publication : 1 avril 2001

Pagination : 679-696

ISBN : 2-7132-1398-3

ISSN : $1252-6576$

Référence électronique

Rolf BINNER, Marc JUNG et Terry MARTIN, « The Great terror in the provinces of the USSR,

1937-1938 ", Cahiers du monde russe [En ligne], 42/2-4 | 2001, mis en ligne le 01 janvier 2007, consulté le 03 septembre 2022. URL : http://journals.openedition.org/monderusse/8474 ; DOI : https://doi.org/ 10.4000 /monderusse. 8474 
chercher : repérer : avancer

Cet article est disponible en ligne à l'adresse :

http://www.cairn.info/article.php?ID REVUE=CMR\&ID NUMPUBLIE=CMR 422\&ID ARTICLE=CMR 4220679

The Great terror in the provinces of the USSR, 1937-1938. A cooperative bibliography

par Rolf BINNER, Marc JUNG et Terry MARTIN

Editions de l'EHESS | Cahiers du monde russe

2001/2-3-4 - Vol 42

ISSN 1252-6576 | ISBN 2713213983 | pages 679 à 696

Pour citer cet article :

-BINNER R., JUNG M. et MARTIN T., The Great terror in the provinces of the USSR, 1937-1938. A cooperative bibliography, Cahiers du monde russe 2001/2-3-4, Vol 42, p. 679-696.

Distribution électronique Cairn pour les Editions de l'EHESS.

(C) Editions de l'EHESS. Tous droits réservés pour tous pays.

La reproduction ou représentation de cet article, notamment par photocopie, n'est autorisée que dans les limites des conditions générales d'utilisation du site ou, le cas échéant, des conditions générales de la licence souscrite par votre établissement. Toute autre reproduction ou représentation, en tout ou partie, sous quelque forme et de quelque manière que ce soit, est interdite sauf accord préalable et écrit de l'éditeur, en dehors des cas prévus par la législation en vigueur en France. Il est précisé que son stockage dans une base de données est également interdit. 


\title{
THE GREAT TERROR IN THE PROVINCES OF THE USSR, 1937-1938
}

\author{
A cooperative bibliography
}

\section{Introduction}

The goal of this bibliography is simple: to draw the attention of specialists to a large body of scattered publications related to the mass operations of the Great Terror in the Soviet provinces. We are professional historians, not bibliographers, and we make no claim to have tracked down every single relevant work. Our major effort was devoted to finding primary documentary sources. The section on secondary sources is much more haphazard. We tried to include primarily works related to the provinces and those based on new archival materials. If your own work does not appear in the list, this is certainly due to an oversight on our part, rather than any calculated slight.

We call this a "cooperative bibliography" because we put it together by asking friends and colleagues in the field of Soviet history to send us citations. We are grateful to the following individuals for their assistance: J. Baberowski (Tubingen), V. Birger (Krasnoiarsk), J. Bone (Chicago), J. Burds (Boston), R. W. Davies (Birmingham), M. Ellmann (Amsterdam), A. Getty (Los Angeles), A. Graziosi (Rome), L. Gvozdkova (Kemerovo), W. Hedeler (Berlin), M. Jansen (Amsterdam), B. Lvin (Washington), R. Manning (Boston), B. McLoughlin (Vienna), N. Okhotin (Moscow), S. Papkov (Novosibirsk), G. Rittersporn (Berlin), N. Rytsk (Moscow), Y. Shapoval (Kyiv), A. Stepanov (Kazan'), E. Topinka (L'viv), V. Vasil'ev (Kyiv), S. Vatlin (Moscow), L. Viola (Toronto), S. Wheatcroft (Melbourne).

We would like to continue to up-date this bibliography. Please send further citations to Marc.Junge@ ruhr-uni-bochum.de 
We begin with a list of the major NKVD decrees related to the mass operations of the Great Terror and continue with a list of primary and secondary sources. We have used the transliteration system of the U.S. Library of Congress throughout.

\section{I}

\section{NKVD decrees, circulars and instructions governing the mass operations of the Great Terror, 1937-1938}

The following is a list of the major NKVD decrees, circulars and instructions that governed the mass operations of the Great Terror. It is almost certainly incomplete since access to the former KGB archive in Moscow is severely limited. We have included those decrees that have been published or referred to in published works. The two most important decrees were prikaz 00447, that governed the campaign targeting former kulaks, criminals and numerous other "former peoples," and prikaz 00485 that served as the paradigm for the various "national operations" of the Great Terror. ${ }^{1}$

Prikaz 00439 (25.07.1937) "Ob operatsii po repressirovaniiu germanskikh poddanykh, podozrevavshikhsia v shpionazhe protiv SSSR."

Prikaz 00447 (30.07.1937) "Ob operatsii po repressirovaniiu byvshikh kulakov, ugolovnikov i dr[ugikh] antisovetskikh elementov."

Tsirkuliar 409 (05.08.1937) "Operatsiia po repressirovaniiu naibolee aktivnykh antisovetskikh elementov [...] v lageriakh."

Tsirkuliar 61 (07.08.1937) [Usilenie bor’by s grabiteliami i ugolovnikami-retsidivistami].

Prikaz 00485 (11.08.1937) "Ob operatsii po repressirovaniiu chlenov 'POV', voennoplennykh pol'skoi armii, perebezhchikov iz Pol'shi, politemigrantov i politobmennykh iz Pol’shi, byvshikh chlenov PPS i drugikh pol'skikh politicheskikh partii."

Prikaz 00486 (15.08.1937) "Ob operatsii po repressirovaniiu zhen izmennikov rodiny."

Tsirkuliar 59190 (16.08.1937) "Operatsiia po repressirovaniiu naibolee aktivnykh kontrrevoliucionnykh elementov [...] v tiurmakh GUGB."

Tsirkuliar 68 (22.08.37) "Ob inostrantsakh."

Prikaz 00593 (20.09.1937) "Ob operatsii po repressirovaniiu byvshikh sluzhashchikh 'Kitaisko-Vostochnoi zheleznoi dorogi i reemigrantov iz Manchzhou-Go' (kharbintsy)."

Prikaz 00693 (23.10.1937) "Ob operatsii po repressirovaniiu perebezhchikov- narushitelei gosgranitsy SSSR.”

Prikaz 00698 (28.10.1937) “O konsul’skikh sviaziakh.”

Memorandum 49990 (30.11.1937) "Latyshskaia operatsiia."

Prikaz 50215 (11.12.1937) “Operatsiia po arestam grekov” [unpublished].

1. Prikaz 00447 is discussed in the essay by Junge and Binner in this volume. Prikaz 00485 has been brilliantly analyzed in N. V. Petrov and A. B. Roginskii, “'Pol'skaia operatsiia' NKVD, 1937-1938 gg.," in Repressii protiv poliakov (Moscow, 1997): 22-43. 
Tsirkuliar 52691 (22.12.37) "O repressiiakh sredi kitaitsev" [unpublished].

Direktiva 17089 (18.01.1938) "Ob operativnykh meropriiatiiakh po eseram" [unpublished].

Prikaz 202 (29.01.1938) "Ob arestakh irantsev" [unpublished].

Direktiva 17231 (14.02.1938) [Ob operativnykh meropriiatiiach po men'shevikam i anarkhistam] [unpublished].

Tsirkuliar 326 (16.02.1938) “O repressiiakh sredi afgantsev” [unpublished].

Prikaz 00606 (17.09.1938) "Ob obrazovanii osobykh troek dlia rassmotreniia del na arestovannykh v poriadke prikazov NKVD SSSR Nr. 00485 i dr[ugikh]" [This prikaz concerns exclusively the national operations, not prikaz 00447].

Tsirkuliar (21.09.1938) zamestitelia narodnogo komissara vnutrennikh del L. Berii o raz"iasnenii primeneniia prikaza 00606.

Postanovlenie P4387 (17.11.1938) Soveta Narodnykh Komissarov SSSR i Tsentral'nogo Komiteta SSSR VKP(b) "Ob arestakh, prokurorskom nadzore i vedenii sledstviia."

Prikaz 00762 (26.11.1938) "O poriadke osushchestvleniia postanovleniia SNK SSSR i TsK VKP(b) ot 17 noiabria 1938 g."

Direktiva 2709 (26.12.1938) NKVD i Prokurora SSSR "Ob otmene reshenii byv. troek NKVD."

In the bibliography we note in bold works where the complete text of NKVD decrees have been published.

II

Documentary publications

We have included in our list of documentary publications numerous "memorial books" (knigi pamiati). These books owe their existence to the persistent efforts of relatives and human rights organizations, above all Memorial, to memorialize the victims of the Great Terror (or of Soviet repression more generally). These books list standard biographical data concerning the victims (birth date and place, profession, nationality, place of residence). Others also provide information concerning social origin, education, Party membership, previous convictions as well as the date of arrest, sentencing (including the article of the criminal code, the sentence itself and the sentencing body) and execution. A drawback of these books is that they almost never include those convicted as ordinary criminals. Many of the memorial books also include extensive valuable documentary materials from local and central KGB archives, as well as autobiographical memoirs from survivors. A nearly complete collection of these memorial books can be found in the library of Memorial-Moscow (Malyi Karetnyi pereulok 12, phone: 200-6506) or in the library of the Sakharov Fund (ul. Semlianoi val. 57, stroenie 6, phone: 923-4401). The adresses of local web sites of Memorial can be found in A. Iutkin, "Sluchainye nakhodki v nesluchainom puteshestvii," Russkaia mysl' (02-08.11.2000), p. 11. The web site for Memorial-Moscow also has an extensive list of memorial books: www.memo.ru 
"Belaia kniga." O deportatsii koreiskogo naseleniia Rossii v 30-40-kh godakh. T. 1. Sost. LI U. Khe. and KIM En Un. (Moscow, 1992).

Belaia kniga. O zhertvakh politicheskikh repressii: Samarskaia oblast’. T. 1-14. Preds. red. soveta POPKOV N. E. (Samara, 1993-2000).

Bol' liudskaia. Kniga pamiati tomichei, repressirovannykh v 30-40-e i nachale 50-kh godov. T. 1 (1991), t. 2 (1992), t. 3 (1992), t. 4 (1994), t. 5 (1999). Sost. UIMANOV V. N. (Tomsk, 1991-1999).

Bol' proshedshaia skvoz' gody. Sost. PAVLOV S. M. (Kemerovo, 2000).

Butovskii poligon. 1937-1938 gg. Kniga pamiati zhertv politicheskikh repressii. T. 1-5. Sost. GOLOVKOVA L. A, LIUBIMOVA K. F. et al. (Moscow, 1997-2001) [Prikazy 00485, 00593 in t. 1].

Chas "chornoho vorona." Narisi (Kirovohrad, 1995).

FEOFANOV IU., "Rasstrel po 1-i kategorii," Izvestiia (03.04.1996): 1, 5 [Politbiuro resolutions "Ob antisovetskikh elementakh" of 24.09.1937, 03.12.1937, 31.01.1938, 17.02.1938, 15.09.1938 and letters from provincial Party and NKVD leaders to TsK, Stalin, and Ezhov asking for higher quotas for repression].

GARROS V., KORENEVSKAIA N. and LAHUSEN T., eds, Intimacy and terror. Soviet diaries of the 1930s (New York, 1995).

GETTY J. A. and NAUMOV O. V., The road to terror. Stalin and the self-destruction of the Bolsheviks, 1932-1939 (New Haven, 1999).

GEVORKIIAN N., "Vstrechnye plany po unichtozheniiu sobstvennogo naroda," Moskovskie novosti, 25 (21.06.1992): 18-19 [List of troika members "po prikazu 00447," Politbiuro resolutions "Ob antisovetskikh elementakh" of 1937/1938, and letters from provincial Party and NKVD leaders to TsK, Stalin, and Ezhov asking for higher quotas for repression].

Gody terrora. Kniga pamiati zhertv politicheskikh repressii. T. 1-2. Sost. SUSLOV A. and GASHEVA N. (Perm', 1998-2000).

GULAG (Glavnoe upravlenie lagerei). 1917-1960. Sost. KOKURIN A. I. and PETROV N. V. (Moscow, 2000) [Prikazy 00447, 00485 and 00486].

GULag v Karelii. Sbornik dokumentov i materialov 1930-1941. Sost. ZHUKOV A. IU., MAKUROV V. G. and PETUKHOVA I. G. (Petrozavodsk, 1992).

GULag v Komi krae, 1929-1956. Sost. MOROZOV N. A. (Syktyvkar, 1997).

GVOZDKOVA L., Prinuditel'nyi trud. Ispravitel'no-trudovye lageria v Kuzbasse (30-50-e gg.). T. 1-2 (Kemerovo, 1994).

Iz bezdny nebytiia. Kniga pamiati repressirovannykh kaluzhan. T. 1-3: A-IA. Sost. KALINICHENKO IU. I., LISIANSKII V.IU. and MONIKOVSKAIA N.P. (Kaluga, 1993-1994).

Iz istorii zemli Tomskoi. 1937. Sibirskii Belostok. Sbornik dokumentov i materialov. Sost. KHANEVICH V. A. (Tomsk, 1998) [Great Terror in a Siberian village with Polish population].

Iz istorii zemli Tomskoi. God 1937. Sbornik dokumentov i materialov. Sost. BONDARENKO A.A., MARKOV V.I. and TRENIN B.P. (Tomsk, 1998) [Includes statistics].

Iz t'my zabveniia: Kniga pamiati zhertv politicheskikh repressii. 1918-1954. T. 1: Rossiiskaia Federatsiia. Astrakhanskaia oblast': A-IA. Sost. VOLODIN E. M. et al. (Astrakhan', 2000). 
Khotelos' by vsekh poimenno nazvat'. Kniga-martirolog. T. 1: A-K. [Khabarovskii krai]. Sost. LAVRENTSOV A. P, TARAN M. M. and BESPALOVA T. G. (n.p. n.d.).

Khotelos' by vsekh poimenno nazvat'... Po materialam sledstvennykh del i lagernykh otchetov GULAGa. Sost. OSIPOVA I. (Moscow, 1993).

Kniga pamiati. Martirolog katolicheskoi tserkvi $v$ SSSR. Sost. CHAPLITSKII B. and OSIPOVA I. (Moscow, 2000).

Kniga pamiati. Posviashchaetsia tagil'chanam - zhertvam repressii 1917-1980-kh godov. Sost. KIRILLOV V. M. (Ekaterinburg, 1994).

Kniga pamiati zheleznodorozhnikov - zhertv politicheskikh repressii 1937-1938 gg., zakhoronennykh na Levashovskom memorial'nom kladbishche. T. 1. Sost. VOL'SKII E. V. (SPb, 2000).

Kniga pamiati zhertv politicheskikh repressii (20-50-e gg.). Sost. DASHINSKII S. N., VORONIN V. V. and NECHUSHKIN V. A. (Murmansk, 1997).

Kniga pamiati zhertv politicheskikh repressii Kalininskoi oblasti. Martirolog 1937-1938. T. 1. Sost. KRAVTSOVA E. I. (Tver', 2000).

Kniga pamiati zhertv politicheskikh repressii Kemerovskoi oblasti. T. 1-2. Sost. GVOZDKOVA L. I. (Kemerovo, 1995-1996).

Kniga pamiati zhertv politicheskikh repressii Kirovskoi oblasti. T. 1-3. Sost. LEGOTIN V. V. et al. (Kirov, 2000).

Kniga pamiati zhertv politicheskikh repressii Kurskoi oblasti. T. 1-3. Sost. VLASOVA L. T., SATALKIN N. V., SOSNOVA L. I. et al. (Kursk, 1996-2000).

Kniga pamiati zhertv politicheskikh repressii $v$ Nizhegorodskoi oblasti. T. 1-2. Sost. GOLUBINOVA A.N., GUSEV M. IU., ZHILTSOV V.I. et al. (Nizhnii Novgorod, 1997-2001).

Kniga pamiati zhertv politicheskikh repressii Novgorodskoi oblasti. T. 1-7. Sost. IVANOV S. A., MAZANKINA N. A. and TRABER N. N. (Novgorod, 1993-1997).

Kniga pamiati zhertv politicheskikh repressii $v$ Orenburgskoi oblasti. Sost. ERMAKOV G. V. (Kaluga, 1998).

Kniga pamiati zhertv politicheskikh repressii. "Osuzhdeny po 58-i..." [Kurganskaia oblast] T. 1 (Kurgan, 2000).

Kniga pamiati zhertv politicheskikh repressii [Respubliki Altai]. T. 1-2. Sost. CHEPKIN P. I., VRAZOVSKAIA O. N. and BURAK T. A. (Gorno-Altaisk, 1996-1998).

Kniga pamiati zhertv politicheskikh repressii Respubliki Bashkortostan. T. 1: $A-V$, t. 2: $G$ I, t. 3: K-M. Sost. VALEEV R. A. et al. (Ufa, 1997, 1999, 2001).

Kniga pamiati zhertv politicheskikh repressii Respubliki Khakasiia. T. 1-2. Sost. ABDIN N. S. (Abakan, 1999-2000).

Kniga pamiati zhertv politicheskikh repressii. Respublika Tatarstan. T. 1: A. Sost. IVANOV A. A. (Kazan', 2000).

Kniga pamiati zhertv politicheskikh repressii Stavropol'skogo kraia. T. 1-11. Sost. KOZUB A. L., USTINOVA M. A. et al. (Stavropol', 1996-2001).

Kniga pamiati zhertv politicheskikh repressi. Sverdlovskaia oblast'. T. 1-2: A-D. Sost. BOCHKAREV M. A. et al. (Ekaterinburg, 1999-2000).

Kniga pamiati zhertv politicheskikh repressii. [Udmurtskoi Respubliki]. Sost. ZHUIKOVA R. A., GASHEV A. I. and LEBEDEV L. M. (Izhevsk, 2001). 
Kniga pamiati zhertv politicheskikh repressii. [Ul'ianovskaia oblast']. T. 1-2. Sost. ZOLOTOV IU. M. (Ul'ianovsk, 1996-2001) [Prikaz 00447, t. 1, p. 766-780].

Kniga pamiati zhertv politicheskikh repressii $v$ Vostochnom Zabaikal'e. T. 1. Sost. VASILEVSKII V. I. (Chita, 2000).

Kniga rasstreliannykh. Martirolog pogibshikh ot ruki NKVD $v$ gody bol'shogo terrora (Tiumenskaia oblast'). T. 1-2. Sost. GOL’DBERG R. S. (Tiumen', 1999).

Kniga skorbi. Rasstrel'nye spiski. T. 1. Sost. BOLTINA V. D. and SHELEVA L. B. (Pavlodar, 1999).

KOKIN S. and PSHENNIKOV O., "Bez stroku davnosti. 'Vikryttia' orhanami NKVD 'Viis'kovo-fashysts'koi zmovi u Chervonii Armii'," Z arkhiviv VUChK-GPUNKVD-KGB, 1/2 (1997); 1/2 (1998); 1/2 (1999); 2/4 (2000).

Koreitsy - zhertvy politicheskikh repressii v SSSR 1934-1938 gg. T. 1. Sost. KU S. (Moscow, 2000).

Krasnodarskii krai v 1937-1941 gg. Dokumenty i materialy. Sost. BELIAEV A. M. et al. (Krasnodar, 1997).

"Krasnoe koleso" pereekhalo i cherez "Rostsel'mash" (khronika terrora 30-kh godov). Kniga pamiati zhertv politicheskikh repressii rabotnikov "Rostsel'masha." Sost. VESEL’NITSKII I. M. (Rostov, 1999).

Kyiv: zhertvy represii. T. 1-2. Sost. ABRAMENKO L. (Kyiv, 1997-1999).

Leningradskii martirolog 1937-1938. T. 1: Avgust - sentiabr' 1937 g.; t. 2: Oktiabr' 1937 goda; t. 3: Noiabr' 1937 goda; t. 4: Dekabr' 1937 goda. Sost. RAZUMOV A. IA. et al. (SPb, 1995, 1996, 1998, 1999) [Résumé of tsirkuliar 61 (t. 1, p. 47-48), prikaz 00439 and 00485 (t. 2), tsirkuliar 59190 (t. 2, facsimile reproduction, Illustration 78-79), prikaz 00593 (t. 3)].

"Limity terroru," Karta, 11 (1993): 8-15 [Prikaz 00447 in Polish translation].

LOSHYTS’KYI O., “'Laboratoriia'. Novi dokumenty i svidchennia pro masovi represii 1937-1938 rokiv na Vinnychchyni," $Z$ arkhiviv VUChK-GPU-NKVD-KGB, 1-2 (1998).

LOSHYTS’KYI O., “'Laboratoriia' - 2: Poltava. Dokumental'ni materialy pro masovi represii v Poltavs'koi oblasti u 1937-1938 rr.," Z arkhiviv VUChK-GPU-NKVD$K G B, 2 / 4$ (2000): 129-178.

Martirolog rasstreliannykh i zakhoronennykh na poligone NKVD “Ob"ekt Butovo" 08.08. 1937-19.10. 1938. Sost. GROIAN T. I., PEREDERIA V. A., EVDOKIMOVA S. I. et al. (Moscow-Butovo, 1997).

"Massovye repressii opravdany byt' ne mogut," Istochnik, 1 (1995): 117-131.

"Materialy fevral'sko-martovskogo plenuma CK VKP(b) 1937 goda," Voprosy istorii, 212, 1992; 2, 5-9, 1993; 2, 6, 8, 10, 12, 1994; 2, 4-8, 10, 1995.

Memorial-Aspekt, 1 (1993) [Prikaz 00593, p. 2].

Memorial-Aspekt, 2-3 (1993) [Prikaz 00486, p. 9].

Narymskaia khronika 1930-1945. Tragediia spetspereselentsev. Dokumenty i vospominaniia. Sost. MAKSHEEVA V. N. (Moscow, 1997).

Ne predat' zabveniiu. Kniga pamiati repressirovannykh $v$ 30-40-e i nachale 50-kh godov, sviazannykh sud’bami s Iaroslavskoi oblast'iu. T. 1-5. Sost. GOLIKOV V. P., KONOPLIN A. V. and ZHOKHOVA G. A. (Iaroslavl', 1993-1998) [T. 2-5 with a new subtitle: Kniga pamiati zhertv politicheskikh repressii, sviazannykh sud'bami s Iaroslavskoi oblast 'iu. T. 4 has Politbiuro resolutions from 1937-1938]. 
Ne predat' zabveniiu: Kniga pamiati zhertv politicheskikh repressii. T.1-10. Sost. KORNEEV N. P. (Pskov, 1996-2000).

Neizvestnaia Kareliia, 1921-1940. Dokumenty spetsorganov o zhizni respubliki. Sost. KLIMOVA A. V., MAKUROV V. G. and FILATOVA A. T. (Petrozavodsk, 1997).

Nimtsi v Ukraini 20-30-ti rr. XX st. Zbirnyk dokumentiv derzhavnykh arkhiviv Ukrainy (Kyiv, 1994).

Odesskii martirolog. Dannye o repressirovannykh Odessy i Odesskoi oblasti za gody sovetskoi vlasti. T. 1-2. Sost. KOVAL'CHUK L. V. and RAZUMOV G. A. (Odessa, 1997-1999) [Includes statistical charts]

OKHOTIN N. and ROGINSKII A., “'Latyshskaia operatsiia' 1937-1938 godov. Arkhivnye kommentarii," "30 Oktiabria”, 4 (2000): 5 [Memorandum 49990].

Organy gosudarstvennoi bezopasnosti SSSR v Velikoi Otechestvennoi voine. Sbornik dokumentov. T. 1: Nakanune. Kniga pervaia (noiabr' 1938 g. - dekabr' 1940 g.). Sost. IAMPOL'SKII V.P. (Moscow, 1995) [Postanovlenie SNK/TsK P4387 and prikaz 00762].

OSIPOVA I., Martirolog katolicheskoi tserkvi v SSSR. Kniga pamiati (Moscow, 2000).

Ostannia adresa. Do 60-richchia solovetskoi tragedii. T. 1-3. Sost. DRACH I., PRYSTAIKO V., PSHENNIKOV O. et al. (Kyiv, 1997-1999).

Ot ChK do FSB. Dokumenty i materialy po istorii organov gosbezopasnosti Tverskogo kraia. 1918-1998. Sost. SMIRNOV V. A., BORISOV A. V. and TSVETKOVA M. V. (Tver', 1998) [Tsirkuliar ot 21.09.1938 o raz'“iasnenii primeneniia prikaza 00606, p. 168-170].

Pam'iat'Bikivni. Dokumenty ta materialy (Kyiv, 2000).

Pamiat'. Zhertvy politicheskikh repressii. Rossiiskaia Federatsiia. Respublika Mordoviia. Sost. SEN'KIN P. E. and SARAEV F. P. (Saransk, 2000) [Prikazy 00439, 00447, 00485, 00593, 00693, 00606, 00762].

Pokaianie: Komi respublikanskii martirolog zhertv massovykh politicheskikh repressii. T. 1-4. Sost. NEVSKII G. V. (Syktyvkar, 1998-2001).

Politicheskie repressii $v$ Kazakhstane v 1937-1938 gg. Sbornik dokumentov. Sost. DEGITAEVA L. D. (Alma-Ata, 1998).

Pomorskii memorial. Kniga pamiati zhertv politicheskikh repressii. T. 1: A-K. Otv. red. SHPERLING IU. M. (Arkhangel’sk, 1999).

Povernuni imena (pro reabilitovani zhertvi politychnykh represii u Zaporiz'kii oblasti). Statti, narysy, portrety, biohrafychni dovidki. Kniha 1 (Kyiv, 1998).

Pravda cherez gody. Stat'i, vospominania, dokumenty. Vyp. 2-4. Sost. BUT O. M., NIKOL'SKYI V. M. and SABINA A. M. (Donetsk, 1998-2000).

Pravda cherez roki. Statti, spohady, dokumenty. Sost. KLIUIEV A. P. et al. (Donetsk, 1995).

"Prikazy NKVD SSSR 1934-1941 gg. Katalog rassekrechennykh dokumentov GARFa." Sost. ZLATKIS IA. M. in: Arkhiv noveishei istorii Rossii. Seriia "Katalogi", T. 5 (Novosibirsk, 1999).

"Rasstrel po raznariadke, ili Kak eto delali bol'sheviki," Trud (04.06.1992): 1/4 [First but incomplete publication of prikaz 00447 with Politbiuro resolution "Ob antisovetskikh elementakh" from July 1937]. 
Rasstrel'nye spiski. Moskva 1937-1941. "Kommunarka”, Butovo. Kniga pamiati zhertv politicheskikh repressii. Sost. ERIMINA L. S. and ROGINSKII A. B. (Moscow, 2000).

Rasstrel'nye spiski. T. 1: Alma-Ata, Alma-Atinskaia oblast'. T.2: Almaty, Almatinskaia, Zhamylskaia oblasti. T. 3: Mangistauskaia oblast'. Sost. ALDANIIAZOVA M., ZHOVTIS A. and IALYMOV N. (Alma-Ata, 1996-1998).

Rasstrel'nye spiski. Vyp. 1: Donskoe kladbishche, 1934-1940. Vyp. 2: Vagan'kovskoe kladbishche, 1926-1936. Sost. TIKHANOVA V. A. (Moscow, 1993-1995).

Reabilitatsiia: Kak eto bylo. Dokumenty Prezidiuma TsK KPSS i drugie materialy. T. 1: Mart 1953-fevral' 1956. Sost. ARTIZOV A. N., SIGACHEV IU. V., KHLOPOV V. G. et al. (Moscow, 2000).

Rekviem: Kniga pamiati zhertv politicheskikh repressii na Orlovshchine. T.1-4. Sost. MOSIAKIN I. IA., ALESHIN A. N. and KABANOV V. V. et al. (Orel, 19941998).

Repressii 30-kh godov v Dagestane. Dokumenty i materialy. Sost. OSMANOV A.I. (Makhachkala, 1997).

Repressii v Arkhangel'ske 1937-1938. Dokumenty i materialy. Sost. MITIN V. A., RADISHEVSKAIA V. A., TITOVA T. V. et al. (Arkhangel'sk, 1999).

"Resheniia osobykh troek privodit' v ispolnenie nemedlenno," Istochnik, 5 (1999): 81-85.

ROZHENKO M. and BOHATS'KA E., Sosni Bikivnia svidchat'. Zlochyn proty liudstva. T. 1 (Kyiv, 1999).

SHAPOVAL IU., PRISTAIKO V., ZOLOTAR'OV V., ChK-GPU-NKVD v Ukraini: osoby, fakty, dokumenty (Kyiv, 1997).

Shifrotelegramma sekretaria TsK KP(b) Turkmenistana o kontrrevoliutsionnoi deiatel'nosti afganskikh poddannykh na nashei territorii ot 23 iiulia 1937, RGANI, f. 89, op. 48, d. 8 [This document is available on microfilm through the Chadwyck-Healey/ Hoover microfilming project].

SHREIDER M. P., NKVD iznutri. Zapiski chekista (Moscow, 1995) [Memoir of the Militia Chief, 1937-1938, from Ivanovo, Novosibirsk and Kazakhstan].

Sistema ispravitel'no-trudovykh lagerei v SSSR, 1923-1960. Spravochnik. Sost. SMIRNOV M. B. (Moscow, 1998).

Soprotivlenie v GULAGe. Vospominaniia. Piśma. Dokumenty. Sost. VILENSKII S. S. (Moscow, 1992).

Sovetskoe rukovodstvo. Perepiska 1928-1941. Sost. KVASHONKIN A. V., KOSHELEVA L. P., ROGOVAIA L. A. et al. (Moscow, 1999).

Spisok rasstreliannykh v gorode Borovichi po resheniiam Osoboi Troiki, avg. 1937 - mart 1938 g. ([Borovichi], 1995).

STEPANOV A. F., Rasstrel po limitu. Iz istorii politicheskikh repressii $v$ TASSR $v$ gody "ezhovshchiny" (Kazan', 1999) [Rich documentation about the repression in Tatarstan 1937-1938, especially about the implementation of prikaz 00447].

STETSOVSKII IU., Istoriia sovetskikh repressii. T. 1-2 (Moscow, 1997).

SUDOPLATOV A. P., Tainaia zhizn’ generala Sudoplatova. Pravda i vymysly o moem ottse. T. 1 (Moscow, 1998): 363-393 [Prikaz 00485 with covering letter from Ezhov "O fashistsko-povstancheskoi, shpionskoi, diversionnoi, porazhencheskoi i terroristicheskoi deiatel'nosti pol'skoi razvedki v SSSR"]. 
Tak eto bylo. Natsional'nye repressii v SSSR 1919-1952 gody. T. 1-3. Sost. ALIEVA S. (Moscow, 1993) [T. 1, p. 253 includes the Politbiuro resolution on continuation of the national operations of 31.01.1938].

Tragediia naroda. Kniga pamiati zhertv politicheskikh repressii Respubliki Marii El. T. 1-2. Sost. KALININ N. M., MITRAKOV A. A. and TOKTAROV N. I. (Ioshkar-Ola, 1996-1997).

Udmurtiia: massovye repressii 1930-1950 godov. Issledovaniia, dokumenty. Sost. MARTYNOVA R. F. and VEDERNIKOVA G. I. (Moscow, 1993).

VASIL'EV, V. IU., KALYTKO S. L., KRAVCHENKO P. M. et al., Politychni represii na Podilli (20-30-i rr. XX st.) (Vinnytsia, 1999).

Velykyi teror na Khmel'nychchyni. Istoriko-kraieznavchyi zbirnyk (svidchennia ta dokumenty) (Khmel'nits'kii, 1997).

Vidomosti pro hromadian, shcho zaznaly politychnykh represii (Mykolaiv, 1993).

Vnutrenniaia opis' dela "Ob antisovetskikh elementakh i sozdanii troek" 09.02.1933 25.05.1943 gg., Rossiiskii Gosudarstvennyi Arkhiv Noveishei Istorii (RGANI), f. 89 , op. 73 , d. 41 [This document is available on microfilm through the Chadwyck-Healey/Hoover microfilming project].

Vozvrashchenie $k$ pravde. (Iz istorii politicheskikh repressii v Tverskom krae v 20-40-e $i$ nachale 50-kh godov). Dokumenty i materialy. Sost. SMIRNOV V. A. and FEOKTISTOV V. A. (Tver', 1995).

VYLTSAN M., "Garantiruetsia vysshaia mera," Trud (02.08.1997): 5 [Documentation on implementation of prikaz 00447].

VYLTSAN M. and DANILOV V., "Primenenie VMN 'nami garantiruetsia'", Nauka i zhizn', 9 (1997): 68-72 [Documents on the implementation of prikaz 00447].

Za nami pridut korabli. Spisok reabilitirovannykh lits, smertnye prigovory $v$ otnoshenii kotorykh privedeny $v$ ispolnenie na territorii Magadanskoi oblasti... Otv. red. ABRAMOV S. V. (Magadan, 1999).

Zabuttiu ne pidliahae. Narysy, spohady, opovidannia (Kherson, 1994).

Zabveniiu ne podlezhit. Kniga pamiati zhertv politicheskikh repressii Omskoi oblasti. T. 1: $A-B$. Sost. ORLOV G. N., SBITNEVA M. A. and CHETVERIKOVA T. G. (Omsk, 2000).

Zabveniiu ne podlezhit. Neizvestnye stranitsy Nizhegorodskoi istorii (1918-1984 gody). T. 2. Sost. GORDEEVA L. P., KAZAKOV V. A. and SMIRNOV V. V. (Nizhnii Novgorod, 1994).

Zabveniiu ne podlezhit. O repressiiakh 30-kh - nachala 50-kh godov v Nizhegorodskoi oblasti. T. 1. Sost. GORDEEVA L. P., KAZAKOV V. A. and SMIRNOV V. V. (Nizhnii Novgorod, 1993).

Zapiska Bykova (Checheno-Ingushskii obkom partii) ob organizatsii troiki v ChechenoIngushskoi ASSR, $\mathrm{N}^{\circ} 115$ ot 10 iiulia 1938 g., RGANI, f. 89, op. 73, d. 147, 11. 1-8 [This document is available on microfilm through the Chadwyck-Healey/Hoover microfilming project].

Zhertvy politicheskikh repressii Irkutskoi oblasti: pamiat' i preduprezhdenie budushchemu. T. 1: A-B. Sost. BOKHANOV P. P. et al. (Irkutsk, 1998).

Zhertvy politicheskikh repressii v Altaiskom krae. T. 1: 1919-1930; t. 2: 1931-1936, t. 3: 1937. Red. TABAREV N. I., RAZGON N. I. et al. (Barnaul, 1998-2001). 
Zhertvy repressii. Nizhnii Tagil 1920-1980-e gody. Sost. KIRILLOV V. M. (Ekaterinburg, 1999).

ZHIROMSKAIA V.B., KISELEV I. N. and POLIAKOV IU. A., Polveka pod grifom sekretno. Vsesoiuznaia perepis’́n naseleniia 1937 g. (Moscow, 1996).

\section{III}

\section{Secondary sources}

ADAMUSHKA U. I., Palitychyia represii 20-50-ykh gadou na Belarusi (Minsk, 1994).

AL’BATS E., Mina zamedlennogo deistviia. (Politicheskii portret KGB) (Moscow, 1992) [With incomplete facsimile reproduction of prikaz 00447].

ALEKSEENKO I. I., Repressii na Kubani i Severnom Kavkaze v 30-e gg. XX veka (Krasnodar, 1993).

BABEROWSKI J., "Auf der Suche nach Eindeutigkeit. Zivilisatorische Mission, Nationalismus und die Ursprünge des Stalinismus in Azerbajdzhan 1828-1941." Habilitationsschrift Universität Tübingen 2000 [Chapter 9 about the Great Terror].

BABEROWSKI J., "'Entweder für den Sozialismus oder nach Archangel'sk'. Stalinismus als Feldzug gegen das Fremde," Osteuropa, 50, 6 (2000): 617-637.

BABEROWSKI J., "Stalinismus als imperiales Phänomen: die islamischen Regionen der Sowjetunion, 1920-1941," in: PLAGGENBORG St., ed., Stalinismus. Neue Forschungen und Konzepte (Berlin, 1998): 113-150.

BABEROWSKI J., "Stalinismus an der Peripherie. Das Beispiel Azerbajdzhan," in: HILDERMEIER M., ed., Stalinismus vor dem Zweiten Weltkrieg. Neue Wege der Forschung (Munich, 1998): 307-335.

BELKOVETS L. P., "Bol'shoi terror" i sud'by nemetskoi derevni v Sibiri (konets 1920kh-1930-e gody) (Moscow, 1995).

BENVENUTI F., "Industry and purge in the Donbass, 1936-1937," Europe-Asia Studies, 45, 1 (1993): 57-78

BEREZHKOV V.I., Piterskie prokurory. Rukovoditeli VChK-MGB 1918-1954 (SPb, 1998): $165 \mathrm{ff}$.

BETTANIN F., Il lungo terrore. Politica e repressioni in URSS, 1917-1953 (Rome, 1999).

BILOKIN' S., Masovyi teror iak zasib derzhavnoho upravlinnia v SRSR (Kyiv, 1999).

BILOKIN S., Mekhanizm bol'shevitskogo nasiliia. Konspekt issledovaniia (Kiev, 2000).

BLITSTEIN P. A., "Selected bibliography of recently published document collections on Soviet history," Cahiers du Monde russe, 40 1-2 (1999): 307-326.

BOTTERBLOEM K.N., “Aspekte der stalinistischen 'Säuberungen' in der russischen Provinz," Jahrbuch für Historische Kommunismusforschung, 1993: 60-81.

BRODSKIJ Ju., Le isole del martirio. Da monastero a primo lager sovietico (n.p., 1998).

BRIUKHANOV B.B. and SHOSHKOV E.N., Opravdaniiu ne podlezhit. Ezhov $i$ ezhovshchina 1936-1938 gg. (SPb, 1998).

BUGAI N. F., Iosif Stalin - Lavrentiiu Berii: "Ikh nado deportirovat'." Dokumenty, fakty, kommentarii (Moscow, 1992).

BUGAI N. F. and GONOV A. M., Kavkaz. Narody v eshelonakh (20-60-e gody) (Moscow, 1998). 
BUGAI N. F., L. Beriia - I. Stalinu: "Soglasno Vashemu ukazaniiu...”(Moscow, 1995).

CHEBOTAREVA V. G., Gosudarstvennaia natsional'naia politika v Respublike nemtsev Povolzh'ia 1918-1941 gg. (Moscow, 1999).

CHENTSOV V. V., Tragicheskie sud'by. Politicheskie repressii protiv nemetskogo naseleniia Ukrainy v 1920-e -1930-e gody (Moscow, 1998).

CHLEWNJUK O., "Partei und NKWD. Die Machtverhältnisse des 'Großen Terrors", (Referat auf der Hamburger Arbeitstagung "Stalinistischer Terror, Massenrepressalien, Gulag" vom 21.-22. Februar 1998).

CHLEWNJUK O. W., Das Politbüro. Mechanismen der Macht in der Sowjetunion der dreißiger Jahre (Hamburg, 1998).

Chorna knyha Ukrainy. Zbirnyk dokumentiv, arkhivnykh materialiv, statei, doslidzhen', ese (Kyiv, 1998).

CHUKHIN I., Kareliia-37: ideologiia i praktika terrora (Petrozavodsk, 1999) [Exhaustive study about the mass operations in Karelia with statistics on the implementation of prikaz 00447 and the "national operations." On page 160 is tsirkuliar 409].

CONQUEST R., The Great Terror. A reassessment (London, 1990).

DANILOV N. N., “'Zaryty, no ne pokhoronenny',” Memorial-Aspekt, 9 (1994): 5.

DOIKOV IU., "Bol'shoi terror v Arkhangel’ske," Russkaia mysl', 4110 (January, 1994).

FITZPATRICK Sh., "How the mice buried the cat. Scenes from the Great Purges of 1937 in the Russian provinces," Russian Review, 52, 3 (1993): 299-320.

FOIGT L. I., Stalinsk $v$ gody repressii. Vospominaniia. Pis'ma. Dokumenty (Novokuznetsk, 1995).

FREEZE G. L., "The Stalinist assault on the parish, 1929-1941," in: HILDERMEIER, M., ed., Stalinismus vor dem Zweiten Weltkrieg. Neue Wege der Forschung (Munich, 1998): 209-232.

FROLOV N., Tragediia naroda. Iz istorii repressii Cheremshanskogo raiona Tatarstana (Kazan', 1999).

GAVRILENKO V. K., Kazn’ prokurora (Abakan, 2000).

GETTY J. A., Origins of the Great Purges: The Soviet Communist Party reconsidered, 1933-1938 (Cambridge, 1985).

GOL’DBERG R., "Slovo i delo po-sovetski. Poslednii iz NKVD,” Rodina, 9 (1998): 85-87.

GRISHAEV V.F., Dvazhdy ubitye ( $K$ istorii stalinskikh repressii v Biiske) (Barnaul, 1999).

GRISHAEV V. F., Reabilitirovany posmertno (k istorii stalinskikh repressii na Altae) (Barnaul, 1995).

GVOZDKOVA L., Istoriia repressii $v$ stalinskikh lagerei $v$ Kuzbasse (30-50-e gg.) (Kemerovo, 1997).

HAGENLOH P.M., "'Socially harmful elements' and the Great Terror," in: FITZPATRICK Sh., ed., Stalinism. New directions (London, 1999): 286-308.

HARRIS J.H., "The purging of local cliques in the Urals region, 1936-1937," in: FITZPATRICK Sh., ed., Stalinism. New directions (London, 1999): 262-285.

HEDELER W., "Gibt es eine 'Schwarzbuchdebatte' in Russland," Sozialismus, 12 (1998): 43-48 [Includes a list of knigi pamiati]. 
HLEVNJUK O.,"Les mécanismes de la 'Grande Terreur' des années 1937-1938 au Turkménistan," Cahiers du Monde russe, 39, 1-2 (1998): 197-208.

ILIC M., "The Great Terror in Leningrad: A quantitative analysis," Europe-Asia Studies, 52, 8 (2000): 1515-1534.

IL’KEVICH N., "Rasstreliany v Viaz'me: novoe o M. N. Goretskom,” Krai Smolenskii, 12 (1994): 129-144.

IL’KEVICH N., "Delo Makedonova.” Iz istorii repressii protiv Smolenskoi pisatel'skoi organizatsii, 1937-1938 g.g. (Smolensk, 1996).

IUNUSOVA A. B., Islam v Bashkirii, 1917-1994 (Ufa, 1994).

IVANOV V. A., Missiia ordena. Mekhanizm massovykh repressii $v$ Sovetskoi Rossii $v$ kontse 20-kh-40-kh gg. (po materialam Severo-Zapada RSFSR) (SPb, 1997).

IVANOVA G. M., Gulag v sisteme totalitarnogo gosudarstva (Moscow, 1997).

IVANOVA T.S., Iz istorii politicheskikh repressii v Iakutii, konets 20-kh-30-e gg. (Novosibirsk, 1998).

Iz istorii spetssluzhb Buriatii. Sost. KHALANOV V. I. and BAZAROV B. V. (Ulan-Ude, 1997).

KANGAS R. D., "Faizulla Khodzhaev: National communism in Bukhara and Soviet Uzbekistan, 1896-1938,” Ph.D. Diss., Indiana University, 1992.

KHAUSTOV V. N., "Deiatel'nost' organov gosudarstvennoi bezopasnosti NKVD SSSR 1934-1941," Diss. dokt. ist. nauk, Moscow, 1997.

KHLEVNIUK O., "Izdaniia 'Memoriala'," Svobodnaia mysl', 7 (1992): 123-132.

KHLEVNIUK O. V., "The reasons for the 'Great Terror': The foreign-political aspect," in: PONS S. and ROMANO A., eds, Russia in the age of wars, 1914-1945 (Milan, 2000): 159-169.

KHLEVNJUK O., Stalin e la società sovietica negli anni del terrore (Perugia, 1997) [This book exists only in Italian].

KHLEVNYUK O., "The objectives of the Great Terror, 1937-1938," in: COOPER J., PERRIE M. and REES E. A., eds, Soviet history, 1917-1953. Essays in honour of R. W. Davies (London, 1995): 158-176.

KIPROV I. A., "Sledstvennoe delo Smolenskoi terroristicheskoi gruppy ob"edinennogo biuro P.S.R. 1937-1938 gg. (ugolovno-protsessual'nyi aspekt)," in: Stalinizm v rossiiskoi provintsii: Smolenskie arkhivnye dokumenty v prochtenii zarubezhnykh i rossiiskikh istorikov. Sost. KODIN E. V. (Smolensk, 1999): 264-275.

KIR'IANOVA E. A., "Sotsial'no-ekonomicheskie i politicheskie protsessy v derevne v 1933 - 1937 godakh (po materialam Moskovskoi, Riazanskoi i Tul'skoi oblastei)," Diss. kand. ist. nauk, Riazan', 1994.

KIRILLOV V. M. and PORSHNEVA O. S., "Istoriia repressii na Urale: Ideologiia, politika, praktika (1917-1980-gg.). Zametki s nauchnoi konferentsii [ot 1012.11.1997]," Otechestvennaia istoriia, 4 (1998): 210-212.

KIRILLOV V. M., "Istoriia repressii na Urale. 1920-e - nachalo 50-kh gg. Na materialakh Nizhnetagil'skogo raiona," Diss. dokt. ist. nauk, Nizhnii Tagil, 1996.

KIRILLOV V. M., Istoriia repressii v Nizhnetagil'skom regione Urala. 1920-e - nachalo 50-kh gg. T. 1: Repressii 1920 - 1930-kh gg., t. 2: Tagillag. 1940-e - nach. 50-kh gg. (Nizhnii Tagil, 1996) [Well-documented study].

KOKURIN A. and PETROV N., "Gulag: Struktura i kadry," Svobodnaia mysl'-XXI. Teoreticheskii i politicheskii zhurnal, 8 , 9, 11, 12 (1999), 1-6 (2000). 
KOSTYRCHENKO G. V. and KHAZANOVYM B.IA., "Konets kar'ery Ezhova," Istoricheskii arkhiv, 1 (1992): 123-131 [Postanovlenie Nr. P4387].

KOTKIN S., Magnetic mountain. Stalinism as a civilization (Berkeley, 1995).

KROPACHEV S., Khronika kommunisticheskogo terrora. Tragicheskie fragmenty noveishei istorii Otechestva. Sobytiia. Masshtaby. Kommentarii. T. 1: 1917-1940 (Krasnodar, 1995).

KUDRIAVTSEV S.V., "Partiinye organizatsii i organy NKVD v period massovykh politicheskikh repressii 1930-kh godov (na materialakh oblastei Verkhnego Povolzh'ia)," Diss. kand. ist. nauk, Iaroslavl', 2000.

KUROMIYA H., Freedom and terror in the Donbas : A Ukrainian-Russian borderland, 1870s-1990s (Cambridge, 1998).

KUZ’MIN S., "Lagerniki (GULAG bez retushi),” Molodaia gvardiia, 4 (1993): 170-212.

LAHUSEN T., "Loin de Moscou. Nouveaux écrits sur l'Extrême-Orient russe de l'ère stalinienne," Revue des Études slaves, 71, 1 (1999): 7-10.

LIKHOLOBOVA Z. G., Stalins 'kyi totalitarnyi rezhym ta politychni represii kintsia 30-kh rokiv v Ukraini (perevazhno na materialakh Donbasu) (Donets'k, 1996).

Lubianka. VChK-OGPU-NKVD-MGB-MVD-KGB 1917-1960. Spravochnik. Sost. KOKURIN A. I. and PETROV N. V. (Moscow, 1997).

MANNING R., Bel'skii raion. $1937 \operatorname{god}($ Smolensk, 1998).

MANNING R., "Massovaia operatsiia protiv 'kulakov i prestupnykh elementov': apogei Velikoi Chistki na Smolenshchine," in: Stalinizm v rossiiskoi provintsii. Smolenskie arkhivnye dokumenty v prochtenii zarubezhnykh i rossiiskikh istorikov. Sost. KODIN E. V. (Smolensk, 1999): 230-254.

MANNING R. T., "The Great Purges in a rural district: Belyi raion revisited," in: GETTY J. A. and MANNING, R. T., eds, Stalinist terror. New perspectives (Cambridge, 1993): 168-197.

MARTIANOV V.E., "Organy NKVD Krasnodarskogo kraia nakanune i v gody Velikoi Otechestvennoi voiny 1937-1945 gg.," Diss. kand. ist. nauk, Krasnodar, 1998.

MARTIN T., "Terror gegen Nationen in der Sowjetunion,” Osteuropa, 50, 6 (2000): 606616.

MARTIN T., The Affirmative Action Empire: Nations and nationalism in the Soviet Union, 1923-1939 (Ithaca, 2001).

MARTIN T., "The origins of Soviet ethnic cleansing," The Journal of Modern History, 70 (1998): 813-861.

McLOUGHLIN B., “'Vernichtung des Fremden': Der 'Große Terror' in der UdSSR 1937/ 38," Jahrbuch für Historische Kommunismusforschung (2000/2001): 50-88.

MIRONENKO A. N. and BEN'KO A. P., Zhertvy stalinskogo terrora predvoennogo piatiletiia na Ukraine (Kyiv - Dniprodzerzhynsk, 1994).

NIKOL'SKYI V., "Ukraintsi Donechchyny, repressirovani v 1937-1938 rr.: sotsiolohychnyi analiz statystyky," Skhid, 3 (1995): 37-45.

NIKOL' SKYI V., "Statystyka politychnykh represii 1937 r. v Ukrains'kyi RSR,” $Z$ arkhiviv VUChK-GPU-NKVD-KGB, 2/4 (2000): 103-112.

NORTHRUP D. T., "Languages of loyalty: Gender, politics and party supervision in Uzbekistan, 1927-1941,” Russian Review, 59 (2000): 179-200. 
NORTHRUP D. T., "Uzbek women and the veil: Gender and power in Stalinist Central Asia," Ph.D. diss., Stanford University, 1999.

OKHOTIN N. and ROGINSKII A., "Iz istorii 'nemetskoi operatsii' NKVD 1937-1938 gg." in: Nakazannyi narod. Repressii protiv rossiiskikh nemtsev. Red.-sost. SHCHERBAKOVA I. L. (Moscow, 1999): 35-75 [Tsirkuliar 68 "Ob inostrantsakh" of 22.08.1937 and prikaz 00698 "O konsul'skikh sviaziakh"].

OSOKINA E., Za fasadom stalinskogo izobiliia. Raspredelenie i rynok $v$ snabzhenii naseleniia $v$ gody industrializatsii, 1927-1941 (Moscow, 1999).

PAL’VANOVA B. P., Tragicheskie 30-e (Ashkhabad, 1991).

PAPKOV S. A., "Lagernaia sistema i prinuditel'nyi trud v Sibiri i na Dal'nem vostoke 1929-1941 gg.," in: Vozvrashchenie pamiati. Istoriko-publitsisticheskii al'manakh. Vyp. 3 (Novosibirsk, 1997): 37-67.

PAPKOV S. A., Stalinskii terror v Sibiri 1928-1941 (Novosibirsk, 1997).

PECHERINA V.F., "Russkie emigranty iz Kitaia i stalinskie repressii v SSSR (19201940-e gg.)," in: Politicheskie repressii na Dal'nem Vostoke SSSR v 1920-1950-e gody. Materialy pervoi Dal'nevostochnoi nauchno-prakticheskoi konferentsii (Vladivostok, 1997): 172-188.

PETROV N., "Tod nach Plansoll: Der operative NKWD-Befehl Nr. 00447" (Referat auf der Hamburger Arbeitstagung "Stalinistischer Terror, Massenrepressalien, Gulag" vom 21.-22. Februar 1998).

PETROV N. V. and ROGINSKII A. B., “'Pol'skaia operatsiia' NKVD 1937-1938,” in: Repressii protiv poliakov i pol'skikh grazhdan. Istoricheskie sborniki "Memoriala." Vyp. 1 (Moscow, 1997): 22-43.

PETROV N. V. and SKORKIN K. V., Kto rukovodil NKVD 1934-1941. Spravochnik (Moscow, 1999).

PETRUSHIN A. A., "My ne znaem poshchady..." Izvestnye, maloizvestnye i neizvestnye sobytiia iz istorii Tiumenskogo kraia po materialam VChK-GPU-NKVD-KGB (Tiumen', 1999).

PLAGGENBORG S., "Gewalt im Stalinismus. Skizzen zu einer Tätergeschichte," in: HILDERMEIER M., ed., Stalinismus vor dem Zweiten Weltkrieg. Neue Wege der Forschung (Munich, 1998): 193-208.

PLATONAU R. and STASHKEVICH M., "Dzve aperatsyi suprats' 'vorahav naroda'," Belaruski histarchny chasopis, 1 (1993) [Statistics on the national operations].

POHL D., "Stalinistische Massenverbrechen in der Ukraine 1936-1953. Ein Überblick," Jahrbuch für Historische Kommunismusforschung (1997): 325-337.

POHL J. O., The Stalinist penal system. A statistical history of Soviet repression and terror, 1930-1953 (Jefferson, NC, 1997).

POHL O., Ethnic cleansing in the USSR, 1937-1949 (Westport, CT, 1999).

POLESHCHIKOV V. M., Za sem 'iu pechatiami: Iz arkhiva KGB [O politicheskikh presledovaniiakh deiatelei Komy kul'tury, sud'bakh politicheskikh zakliuchennykh v lageriakh Komi ASSR] (Syktyvkar, 1995).

Politicheskie repressii na Dal'nem Vostoke SSSR v 1920-1950-e gody. Materialy pervoi Dal'nevostochnoi nauchno-prakticheskoi konferentsii (Vladivostok, 1997).

Politicheskie repressii v Khakasii i drugikh regionakh Sibiri (1920-1950 g.). Materialy mezhregional'noi nauchno-prakticheskoi konferentsii, prokhodivshei 20.10.2000 v Abakane (Abakan, 2001). 
Pravda i tol'ko pravda. "Belye piatna" nashei istorii (Baku, 1991).

Problemy istorii repressivnoi politiki na evropeiskom Severe Rossii 1917-1956: Vserossiiskaia nauchnaia konferentsiia 8-11 noiabria 1993 g. Otv. red. MOROZOV N. A. (Syktyvkar, 1993).

Problemy sozdaniia edinogo elektronnogo banka dannykh zhertv politicheskikh repressii $v$ predelakh SSSR. Sbornik dokladov uchastnikov mezhdunarodnoi nauchno-prakticheskoi konferentsii (Nizhnii Tagil, 2000).

Provintsial'naia Cheka. Sbornik statei i materialov (Khar'kov, 1994).

RASSKAZOV L. P., "VChK-GPU-OGPU-NKVD v mekhanizme formirovaniia i funktsionirovaniia politicheskoi sistemy sovetskogo obshchestva (1917-1941)," Diss. dokt. iuridicheskikh nauk, $\mathrm{SPb}, 1994$.

RAZUMOV A. IA., "Kniga pamiati 'Leningradskii martirolog. 1937-1938' kak istochnik dlia sinodika Vologodskoi eparkhii," in: Regional'nye aspekty istoricheskogo puti pravoslaviia: arkhivy, istochniki, metodologiia issledovanii. Istoricheskoe kraevedenie $i$ arkhivy. Vyp. 7 (Vologda, 2001): 454-460.

Reabilitovani istoriieiu (Kyiv-Poltava, 1992).

RITTERSPORN G. T., Stalinist simplifications and Soviet complications: Social tensions and political conflicts in the USSR, 1933-1953 (Chur, 1991).

RITTERSPORN G. T., “'Vrednye elementy', 'opasnye men'shinstva' i bol'shevistskie trevogi: massovye operatsii 1937-1938 gg. i etnicheskii vopros v SSSR," in: $V$ sem'e edinoi. Natsional'naia politika partii bol'shevikov i ee osushchestvlenie na Severo-Zapade Rossii v 1920-1950-e gody. Red. VIKHAVAINEN T. and TAKALA I. (Petrozavodsk, 1998): 99-122.

ROSIN V.IU., Iz"iato pri areste. O neizvestnykh faktakh deiatel'nosti NKVD Ukrainy (Kyiv, 1992).

SAMOSUDOV V. M., Bol'shoi terror v Omskom Priirtysh'e 1937-1938 (Omsk, 1998) [Includes a complete list of troika-sessions in Omsk. Extracts from troika potocols of Omsk can be foun under: http://www.ic.omskreg.ru/ protocol].

SANUKOV K., "Stalinist terror in the Mari Republic. The attack on 'Finno-Ugrian bourgeois nationalism'," The Slavonic and East-European Review, 4 (1996): 658-682.

SANUKOV K. N., Iz istorii Marii El: Tragedia 30-kh godov (Ioshkar-Ola, 2000).

SHAPOVAL IU. I., "Stalins'kyi teror u svitli novovyiavlenykh arkhivnykh dokumentiv Sluzhby bezpeky Ukrainy," in: Ukrains 'ke arkhivoznavstvo: istoriia, suchasnyi stan ta perspektyvy (Kyiv, 1997).

SHAPOVAL IU. I., Ukraina 20-50-kh rokiv: storinky nenapysanoi istorii (Kyiv, 1993).

SHAPOVAL J., "NKVD-Terror in der Ukraine" (Referat auf der Hamburger Arbeitstagung "Stalinistischer Terror, Massenrepressalien, Gulag" vom 21.-22. Februar 1998).

SHAPOVAL Y., "The tragic fate of Iuliian Bachynsky," Journal of Ukrainian Studies, 23, 1 (1998): 25-39.

SHAPOVAL YU. I., Liudyna i systema: Shtrykhy do portreta totalitarnoi doby v Ukraini (Kyiv, 1994).

SHEARER D. R., "Crime and social disorder in Stalin's Russia. A reassessment of the Great Retreat and the origins of mass repression," Cahiers du Monde russe, 39, 1-2 (1998): 119-148.

SHKAROVSKII M. V., CHEREPENINA N. IU. and SHIKER A. K., Rimsko-katolicheskaia tserkov’ na Severo-Zapade Rossii v 1917-1949 (SPb, 1998): 211-290. 
SHYTIUK M. M., "Masovi represii na terrytorii Mykolaivshchyny (30-ti rr. XX st.)," Ukrains 'kyi istorychnyi zhurnal, 1 (1998): 94-98.

SOKOLOV A., "Ezhovshchina," in: Vlast' i obshchestvo v SSSR: politika repressii (20 40-e gg.). Sbornik statei. Sost. DMITRENKO V.P., KULIKOVA G. B. and IARUSHINA L. V. (Moscow, 1999): 265-285.

Stalinizm $v$ rossiiskoi provintsii. Smolenskie arkhivnye dokumenty $v$ prochtenii zarubezhnykh i rossiiskikh istorikov. Red. KODIN E. V. (Smolensk, 1999).

STANKOVSKAIA G., "Kak delali 'vragov naroda'," in: Gody terrora. Kniga pamiati zhertv politicheskikh repressii. Sost. SUSLOV A. B. and GASHEVA N. (Perm', 1998): 92-108.

STEPANOV A., "Rasstrel po limitu," in: Volia, 6-7 (1997): 106-113.

STEPANOV A. F. and ZEMSKOV V. N., "O nekotorykh problemakh 'Bol'shogo Terrora' 1937-1938 godov," Otechestvennaia istoriia, 1 (2000): 197-205.

STROMBERG A.G., "Rasstrelometriia. Politicheskie repressii v Tomskoi oblasti 1928-1953," in: Totalitarizm i totalitarnoe soznanie. Sbornik statei i materialov (Tomsk, 1996): 70-89.

SULTANBEKOV B.F., "Massovye repressii 30-kh. Zakony, prikazy, ispolniteli," in: Mertvym ne bol'no. Bol'no zhivym (Kazan', 2000): 6-33.

SUTURIN A., Delo kraevogo masshtaba. O zhertvakh stalinskogo bezzakoniia na Dal'nem Vostoke (Khabarovsk, 1991).

TABACHNIK D. and ROZTAL'NII V., "Stalins'kii teror. Ukraina naprikintsi tridtsiatikh," Kyiv, 2 (1989): 140-157.

TAKALA I., "Natsional'nye operatsii OGPU/NKVD v Karelii," in: $V$ sem'e edinoi. Natsional'naia politika partii bol'shevikov i ee osushchestvlenie na Severo-Zapade Rossii v 1920-1950-e gody. Red. VIKHAVAINEN T. and TAKALA I. (Petrozavodsk, 1998): 161-206.

TEPLIAKOV A. G., "Personal i povsednevnost' Novosibirskogo UNKVD v 1936-1946," Minuvshee, 21 (1997): 240-293.

THURSTON R. W., Life and terror in Stalin's Russia 1934-1941 (New Haven, 1996).

"Travaux récents sur l'histoire des répressions de l'ère stalinienne en Extrême-Orient. Bibliographie sélective," Revue des Études slaves, 71, 1 (1999): 11-16.

TUGUZHEKOVA V. N. and KARLOV S. V., Repressii v Khakasii (Abakan, 1998).

UIMANOV V. N., Repressii. Kak eto bylo... (Zapadnaia Sibir' v kontse 20-kh - nachale 50-kh godov) (Tomsk, 1995).

VATLIN A., "Die Erfindung des 'Bundes polnischer Patrioten' (1937-1938). Aus den Archiven des NKVD," Forum für osteuropäische Ideen-und Zeitgeschichte, 2, 1 (1998): 116-139.

Veroi i pravdoi. FSB. Stranitsy istorii (Iaroslavl', 2001).

VESELAIA G., "Troika postanovila: Rasstreliat'," Volia, 2-3 (1994): 21-79.

VINOGRADOV V., Genrikh Iagoda. Narkom vnutrennikh del SSSR. General'nyi komissar gosudarstvennoi bezopasnosti. Sbornik dokumentov (Kazan', 1997).

VINOGRADOV V., Karel'skoe "delo." Delo o t. n. "Karel'skoi burzhuazno-natsionalisticheskoi, terroristicheskoi, kontrrevoliutsionnoi organizatsii”"(Tver', 1991).

Vlast' i obshchestvo $v$ SSSR: politika repressii (20-40-gg.) Sbornik statei. Sost. DMITRENKO V. P., KULIKOVA G. B. and IARUSHINA L. V. (Moscow, 1999). 
$V$ sem’e edinoi. Natsional'naia politika partii bol'shevikov $i$ ee osushchestvlenie na Severo-Zapade Rossii v 1920-1950-e gody. Red. VIKHAVAINEN T. and TAKALA I. (Petrozavodsk, 1998).

VYLTSAN M., "Repressii protiv krest'ian. 30-gody," in: Vlast' i obshchestvo v SSSR: politika repressii (20-40-gg.). Sbornik statei (Moscow, 1999): 236-265.

WEINBERG R., "Purge and politics in the periphery: Birobidzhan in 1937," Slavic Review, 52, 1 (1993): 13-27.

WERTH N., "Logiques de violence dans l'URSS stalinienne," in: ROUSSO H., ed., Stalinisme et nazisme. Histoire et mémoire comparées (Bruxelles, 1999): 99-128.

WERTH N., "Un État contre son peuple. Violences, répressions, terreurs en Union soviétique," in: COURTOIS S., WERTH N. and PANNÉ J.-L. et al., Le livre noir du communisme. Crimes, terreur, répression (Paris, 1997): 43-295.

WHEATCROFT S. G., "The scale and nature of Stalinist repression and its demographic significance: On comments by Keep and Conquest," Europe-Asia Studies, 52, 6 (2000): 1143-1159 [Discussion of data on arrests, sentences and executions from Tomsk oblast' in Western Siberia].

ZAKHAROV V. D., "O politicheskikh repressiiakh 30-kh godov (po dokumentam Komi RGA OPDF)," in: Otechestvennye arkhivy, 1 (1998): 89-92.

ZAKHAROVA G., Mariupol'skaia tragediia (Mariupol', 2000).

ZOLOTAR'OV V. A. and SHAPOVAL IU. I., "V. A. Balits'kii: Na shliakhu do pravdy pro n’oho," Ukrains 'kyi istorychnyi zhurnal, 6 (1993): 50-63; 7/8 (1993): 53-69.

ZOLOTAR'OV V. A. and SHAPOVAL IU. I., "Kolyvan' u provedenni linii partii ne bulo: Storinky biohrafii K.M. Karlsona - zastupnyka narkoma vnutrishnikh sprav URSR,” Ukrains ’kyi istorychnyi zhurnal, 1 (1996). 